\title{
Magnetic resonance imaging and spectroscopy: how useful is it for prediction and prognosis?
}

\author{
Barrie Condon
}

Received: 25 March 2011 / Accepted: 3 May 2011 / Published online: 25 May 2011

(C) European Association for Predictive, Preventive and Personalised Medicine 2011

\begin{abstract}
MRI/MRS can produce information on over 40 physico-chemical parameters regarded as biomarkers of structural, functional or metabolic significance. Though of undisputed worth in the detection of macroscopic lesions or of metabolic derangements, MRI's use in prognosis and prediction has not been so extensively studied. Serial studies can be performed to show early pre-clinical changes in biomarkers caused by disease progression or therapy, such as the adverse effect on heart function of certain cancer therapies. It can utilise various haemodynamic measures to predict the evolution of stroke and so help justify certain interventions. Changes in cerebral metabolite concentrations or the volumes of brain sub-structures can be used as objective measures of drug response in psychiatric conditions. However care must be exercised as MR can sometimes be considered 'too sensitive' as it often detects real abnormalities even in asymptomatic volunteers, the actual predictive significance of which have yet to be fully assessed.
\end{abstract}

Keywords MRI $\cdot$ MRS $\cdot$ Prognosis $\cdot$ Prediction

\section{MRI and MRS}

Magnetic Resonance Imaging (MRI) is a non-invasive technique which can provide information on $\mathrm{T} 1$ and $\mathrm{T} 2$ (NMR relaxation parameters which change with disease and which provide the main diagnostic images in MRI), proton density (an index of the water content of tissue),

B. Condon $(\bowtie)$

MRI Unit, Institute of Neurological Sciences,

Glasgow G514TF, UK

e-mail: barrie.condon@glasgow.ac.uk blood flow in the macrovasculature using MR angiography (MRA), perfusion of blood through the microvasculature of tissues and organs (perfusion weighted imaging, PWI), molecular diffusion based images showing preferred directions of free diffusion (diffusion weighted imaging DWI or diffusion tensor imaging DTI), relative proportions of bound and unbound water (magnetisation transfer contrast, MTC) and localised tissue temperature. The spatial resolution is typically $1 \mathrm{~mm}^{3}$ though isotropic resolutions down to 50 microns can be obtained in certain circumstances. Exquisite contrast between tissues and lesions means the technique can also supply high quality volumetric information on organs and tissues and how they change with disease and treatment.

A multiplicity of pulse sequences can be used in MRI, the resulting signal from which will be affected by all the above parameters. However sequences can be 'weighted' towards one or other of these. High frequency serial studies of change in these various parameters are also readily possible with MRI because it does not use ionising radiation.

Magnetic Resonance Spectroscopy (MRS) is a technique which can provide information on tissue chemistry noninvasively. Though information can be obtained in image form the resolution is very poor $\left(1000 \mathrm{~mm}^{3}\right.$ typically) and spectral quality is also limited. Generally therefore studies take the form of acquiring higher quality spectra from single volumes (voxels) of $8000 \mathrm{~mm}^{3}$ or so, i.e. no images are produced. Metabolites which are revealed include Nacetyl aspartate (NAA, thought to be a neuronal marker), Choline-containing compounds (Ch thought to be a marker of cell membrane turnover), lactate (a product of anaerobic glycolysis), phosphocreatine and adenosine triphosphate (PCr and ATP, measures of energy metabolism). Estimates of intracellular $\mathrm{pH}$ can also be made using 31-phosphorus 
based MRS. Quantification in MRS usually takes the form of relative spectral peak areas compared to reference metabolites which are considered not to vary significantly with most pathologies eg creatine containing compounds (Cr). Absolute measures of metabolite concentration can also be made usually with reference to the absolute concentration of unbound water in the tissue which, as an approximation, is assumed not to vary markedly with disease.

MRI and MRS can yield information on over 40 physico-chemical variables. Much of the past 30 years since the technique was introduced clinically have been spent developing techniques for refining estimates of these variables and also in establishing their clinical or research significance.

MRI and MRS are used primarily in the detection and localisation of macroscopic lesions or metabolic derangements but they are being used increasingly as a means of prediction regarding the evolution of disease and as a guide for making interventions.

\section{Prediction and prognosis}

\section{Cancer}

Monitoring chemotherapeutic response in breast cancer is important both as a guide to alter therapy if it is ineffective but also in assessing the critical effects on the organs of these toxic medicines.

Many treatments options are now available, and with in vitro methods of limited reliability in determining chemotherapeutic response [1], as indeed are mammography and echo [2], researchers have applied MRI techniques which are usually based on measures of tissue perfusion (PWI). Loo [3] investigated parameters associated with PWI to predict which tumours with neoadjuvant chemotherapy would achieve remission. PWI is a technique which departs from the usually entirely non-invasive nature of MRI and MRS in that it requires the intravenous injection of a paramagnetic contrast agent. Tumours cause increased vascular permeability [4] allowing contrast material into the tissue. This alters both $\mathrm{T} 1$ and $\mathrm{T} 2$ relaxation times and the changing signal can yield a plethora of parameters, for example time to initial enhancement, time to maximum enhancement, extent of enhancing tissue, relative degree of enhancement. Figure 1 shows an example of an area of enhanced signal in a patient with breast cancer.

Additionally measures of tumour size were made. The group found that the parameter which gave the most significant correlation $(p<0.00001)$ with final histopathology was based on the changes in the largest diameter in the region of late signal enhancement before and during

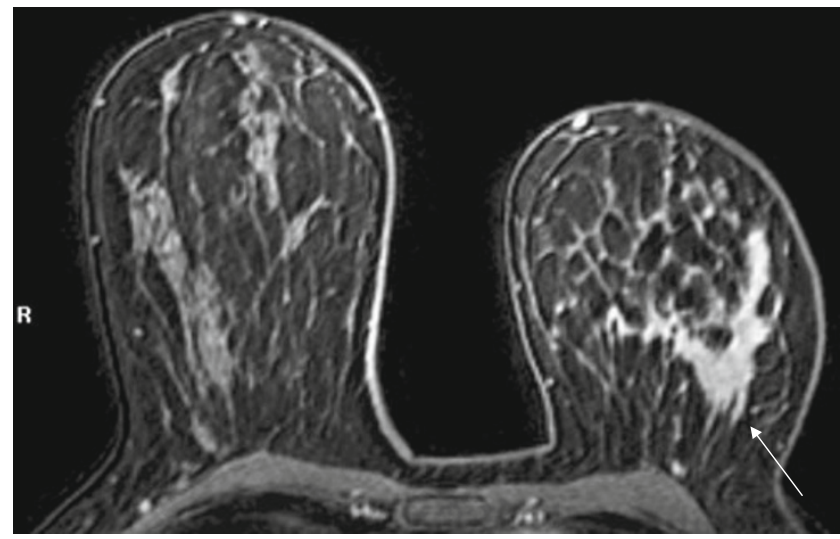

Fig. 1 Breast tumour enhancement following contrast administration. The bright areas (arrowed) show signal change due to the increased vascular permeability within the tumour allowing paramagnetic contrast agent into the tissue

chemotherapy. They found that a reduction of less than $25 \%$ during therapy was associated with residual disease at pathology after therapy. The authors consider this parameter a combination of morphology (ie the extent) and the kinetics (late enhancement) of tumour vasculature. In clinical practice, the MRI response prediction test may offer the oncologist an objective tool of high specificity to tailor the chemotherapy for each individual patient.

Generally size is a good predictor in breast tumours. In a study by Cheung [5], who evaluated 33 patients with serial MRI, it was found that all complete responders had a marked early size reduction of more than $45 \%$.

Tumour size is also, as one would intuitively expect, predictive of survival in other organs such as the brain with recurrent malignant glioma. It should be noted however that previous international standards for measuring tumour size were based on uni- or bi-dimensional measures of tumour (WHO and RECIST) however these were found not to have a significant association with overall survival as they did not adequately assess tumour size in recurrent malignant glioma [6]. Only 3D estimates of volume size were found to be predictive of survival. Using only one or two orthogonal diameters to assess tumour volumes is a hangover from the days when plain $\mathrm{x}$-rays were used in tumour assessment. With the high spatial resolution routinely available with MRI or CT full $3 \mathrm{D}$ volumetric analysis should always be used.

As well as predicting the evolution of disease MRI can be useful in the early detections of adverse effects of therapy. Chemotherapeutic agents such as Trastuzumab for breast cancer can have cardiotoxic effects and it is necessary to serially evaluate left ventricular function for early signs of deterioration. Cardiac MR has become the gold standard for this [7] as it is non-invasive and does not use ionising radiation, as opposed to the Nuclear Medicine based multiple gated acquisition approach and is also more 
accurate than echo based techniques in showing early change in function [7].

However the primary disadvantage of MR is that it usually takes minutes to acquire image sets, which is a problem when imaging a rapidly moving structure like the heart. In order to overcome the blurring effects of motion image acquisition can be gated with respect to the ecg signal so images are built up by interrogating the heart at the same point in its cycle over multiple cycles. By acquiring subsequent images at different points in the heart cycle a cumulated image set can be acquired showing the heart expanding and contracting, from which volumetric estimates of ejection fraction and heart wall motion can be obtained (see Fig. 2). The high level of accuracy and
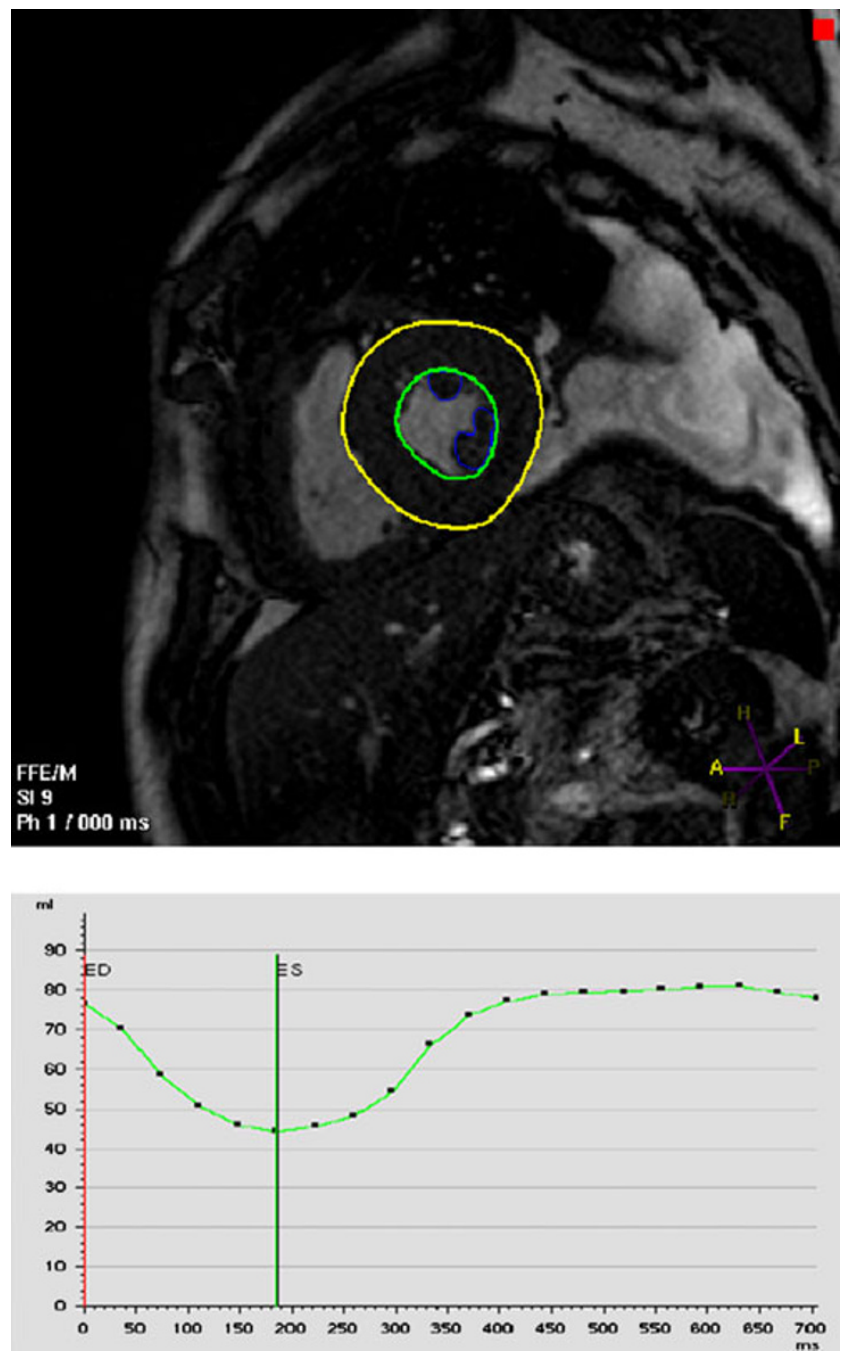

Fig. 2 Image of the left ventricle (LV) at one point in the cardiac cycle. The inner region defines the blood pool, the area between the two regions defines the heart muscle. By delineating the blood pool in each image and by integrating these areas across all the slices which contain the ventricle, will yield the volume of the LV. From these volumes at the time points reflecting diastole and systole the ejection fraction $(\mathrm{EF})$ of the $\mathrm{LV}$ can be calculated. Serial studies can detect early deterioration of $\mathrm{EF}$ due to cardiotoxicity of some cancer drugs reproducibility afforded by cardiac MR (CMR) means it is the best modality for assessing any serial changes in ejection fraction due to the cardiotoxic effects from the therapy.

Stroke

MR is involved in more acute forms of prediction in the field of stroke. Following a stroke some brain tissue will be irrecoverable (the ischaemic zone) but will often be surrounded by a zone where perfusion is reduced and, if blood flow is not restored, over the coming hours will be incorporated into the ischaemic zone. If applied early enough, usually within 6 hours or so of the stroke, thrombolytic therapies can help restore tissue perfusion in this 'penumbral zone' and prevent the growth of the ischaemic zone and hence reduce the level of subsequent disability. Not only can such a measure provide evidence for resorting to thrombolytic therapy but it has been suggested that the reason many trials for new stroke drugs have apparently failed was because patients selected may, for example, not have had significant recoverable tissue to begin with [8].

MRI provides a number of means of defining these zones and hence providing data for clinicians to make informed therapeutic decisions. Within the first few hours of stroke the usual T1 and T2 weighted MR images will not show a lesion, however if the sequences are weighted towards free molecular diffusion (DWI) the lesion is evident within minutes of the event. The accepted explanation is that normal brain cells are surrounded by extracellular water in which there is more or less free diffusion of the water molecules. However under conditions of ischaemia the cells quickly swell up, presenting increasing barriers to this free diffusion. By sensitising our pulse sequences to diffusion this produces a signal change in the ischaemic zone which is readily visible (see Fig. 3).

Perfusion weighted imaging (PWI) will generally reveal a bigger lesion area. In PWI paramagnetic contrast is injected intravenously and images are dynamically taken over the brain to detect the arrival of the bolus which causes signal changes in the perfused tissue. Various measures can be applied to the signal/time curve to extract parameters such as mean time of arrival, blood volume and blood flow. These parameters show the area where perfusion is reduced. The difference in area between the (generally) larger PWI revealed lesion and the DWI revealed lesion represents potentially salvageable tissue. The larger the mismatch the greater the evidence for thrombolytic intervention. (Fig. 3).

The PWI/DWI technique, and indeed the above explanation of it, are however approximate. There is some histological evidence that neither DWI nor PWI accurately 
Fig. 3 DWI (a) and PWI (b) images in stroke. The arrow in the DWI image points to an area which is considered irrecoverable. The arrows in the PWI image point to areas where perfusion has been affected by the stroke but which are still potentially salvageable

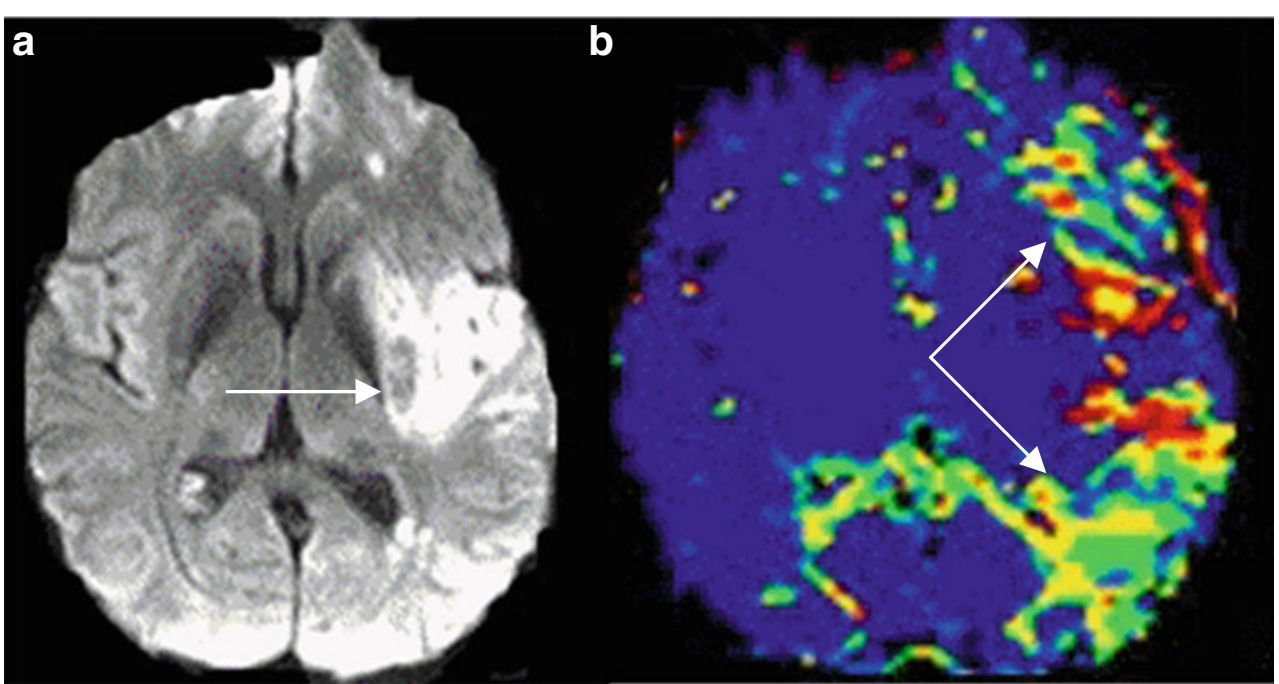

reflect the zones they are purported too and indeed it is occasionally possible to find DWI areas seemingly larger than PWI [9]. In response to this another MRI based technique has been developed which looks at the effects of metabolism rather than blood perfusion. This is the Glasgow Oxygen Level Dependent contrast (GOLD) technique and makes use of the different magnetic properties of oxy and de-oxyheamoglobin [10]. A baseline scan is performed and then repeated but with the patient breathing $100 \%$ oxygen (the 'oxygen challenge'). Where tissue is normally metabolising this extra oxygen reduces the amount of the more magnetic deoxyheamoglobin causing a signal increase. However where perfusion is reduced but the tissue is still metabolising the oxygen extraction fraction is increased producing a higher proportion of oxy- to de-oxyhaemoglobin causing an even higher signal change than in normally metabolising tissue. Ischaemic tissue where there is no metabolism will produce no signal change with the oxygen challenge. It is felt that these criteria based on metabolism may therefore better delineate the ischaemic and penumbral zones.

\section{Alzheimers Disease (AD)}

The high resolution and high contrast afforded by MRI allows careful delineation of brain structures from which volume estimates can be made. Structures such as the hippocampus (see Fig 4), caudate and putamen are of particular interest in this condition. Indeed a meta-analysis [11] showed annualised hippocampal volume reductions of $1.4 \%$ in normals whilst $4.6 \%$ in those with Alzheimers disease. Tabrizi [12] showed longitudinal change over 12 months in caudate and putamen volumes, in individuals many years from predicted disease onset thus showing the feasibility of obtaining quantifiable endpoints for future trials.
Changes in total brain, ventricular, hippocampal and temporal volumes have been shown by many studies to correlate with changes in cognitive performance. In normal ageing whole brain atrophy is usually $0.7 \%$ or less, whilst in $\mathrm{AD}$ it may be typically $1.4-2.2 \%$ with group differences in rate evident over 3 years before diagnosis of $\mathrm{AD}$ [13].

These and other studies are showing predictive differences in groups of patients, but are they accurate and reproducible enough to provide diagnostic predictions for individuals? MRI has long been used to show that all brains show atrophy, and CSF spaces show enlargement, with ageing, however there are a number of sources of error which must be considered. When comparing groups or individuals one must normalise all volume figures with respect to the intracranial cavity volume as brain size can easily vary by $50 \%$ even in the adult normal population

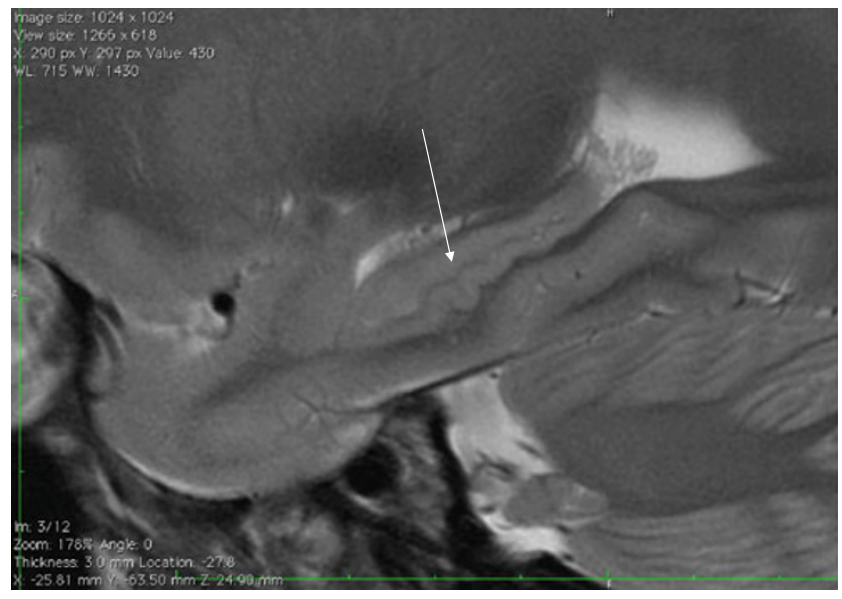

Fig. 4 Sagittal image showing the hippocampus (arrowed). Modern high field MRI provides such exquisite contrast and resolution that not only the volume of the hippocampus, but also its sub-volumes (subfields) can be estimated. The hippocampus is also often the site of investigation for MRS in psychiatric conditions 
[14]. Areas such as the hippocampus are small and difficult to define and automated and semi-automated techniques for volume delineation are prone to significant error.

Nevertheless atrophy of medial temporal structures occur years before symptoms appear at least on a group basis. At the mild cognitive impairment stage such atrophy can be considered a partially validated diagnostic marker [15]. However even at the later stage of $\mathrm{AD}$ only $81 \%$ of the disease group could be correctly identified as having the disease but in the age matched control group only $67 \%$ could be correctly identified as not having the disease[16] making it of limited use for individual diagnosis.

This has led some to the suggestion that combination with other markers such as CSF biomarkers can be more diagnostically accurate [17]. It is hoped that standardisation of technical parameters such as pulse sequence and tissue volume definition criteria will reduce this overlap. Indeed it has been argued that at least some of the overlap is due to imaging of age matched 'controls' who will subsequently develop AD.

\section{Multiple sclerosis}

Complex models have been constructed to try to predict the course of multiple sclerosis. The presence of lesions evident on T2 weighted images (see Fig. 5) can be found in $95 \%$ of patients with clinically definite MS however similarly appearing abnormalities can be found in a number of other diseases but also in healthy volunteers, especially in those who are older. Other factors such as the minimum number of such lesions, position of lesions, size

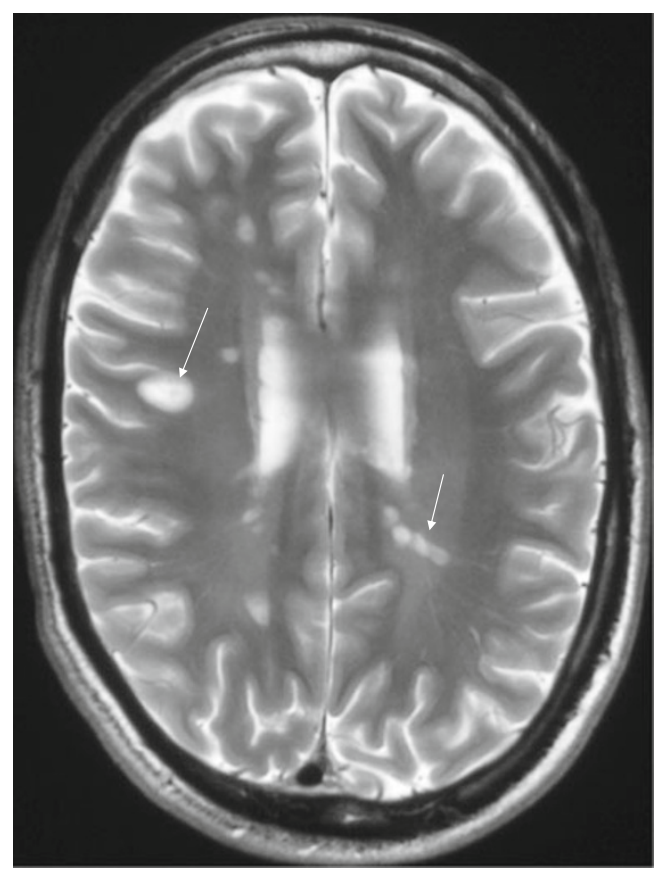

Fig. 5 Image showing a number of white matter lesions in an MS patient and appearance of lesions have been used in combination to 'tune' the technique as regards predicting 'conversion' to clinically definite multiple sclerosis. Sensitivity's of between 80 and $90 \%$ are typical but specificities may be as low as $54 \%$ [18].

However more simple models can have their place, for example Prosperini [19] showed that developing new high signal $\mathrm{T} 2$ lesions during therapy with beta interferon was a good predictor of poor long term response to the treatment with a 10-fold augmented risk for each new lesion. Whilst this might appear intuitively obvious, making the simple assumption that one could therefore also use the appearance of new lesions as a predictor of relapse in untreated MS patients was not however corroborated by the study of Petkau [20] who concluded that such new lesions could not be used as a surrogate outcome for MS relapses.

Psychiatric disorders

A number of parameters measureable by MRI have been applied to various psychiatric conditions, for example to try to predict response to therapies for depression. This is because only about a half of patients finally respond to treatment. During the intervening time before symptom remission (or lack of it) become evident, they will be exposed to risk due to the nature of their condition. Chen [21] found that increased grey matter volumetric measures of the anterior cingulate could provide a useful predictor of antidepressant treatment response.

As with MRI the vast majority of papers on MRS and psychiatric diseases have concerned themselves with diagnosis rather than prediction or prognosis. Block [22] found that low baseline NAA and Cho levels were predictive of positive treatment response for patients with major depressive disorder. These subsequently increased with treatment. Spectra were obtained from the hippocampus and treatment was by citalopram and nortriptyline.

That the situation is more complex than this study would suggest is indicated by the study of Kado [23] which found that patients with depressive psychosis (geriatric) with lower NAA/Cr ratios (which generally would be regarded as indicative of reduced NAA as $\mathrm{Cr}$ is less variant with disease) were less responsive to treatment than those with higher ratios. Spectra were obtained from deep white matter of the frontal lobe bilaterally. A tricyclic antidepressant and a tetracyclic antidepressant were used as the first and second choices for treatment, respectively.

In patients with a high risk of developing schizophrenia significant reductions in the amount of NAA are found in the thalamus [24]. However this is not a specific measure in itself as many conditions, lesional as well as psychiatric such as depression, cause reductions in this. Keshavan [25] found 
in young first degree relatives of schizophrenic patients reduced NAA in the caudate compared to healthy controls.

Jessen [26] reported that in subjects at risk of schizophrenia who subsequently converted had a higher $\mathrm{Cho} / \mathrm{Cr}$ and a lower $\mathrm{NAA} / \mathrm{Cho}$ ratio in the anterior cingulate gyrus compared with non-converters. They concluded that the reduction of the neuronal marker NAA in the left prefrontal lobe and the anterior cingulate gyrus may represent a vulnerability indicator for schizophrenia in at-risk subjects, while elevated Cho in the anterior cingulate gyrus may be a predictor for conversion from the prodromal state to the full disease.

It should be noted that all the above studies show only group differences. The innate signal to noise variations in spectra from individual patients can be used to diagnose disease which causes large scale metabolic derangements (eg tumour, Canavan's disease) but will swamp subtle differences in NAA found in these psychiatric conditions. MRS is therefore a long way from being diagnostic of psychiatric conditions in the individual. Even in group studies minimum sample sizes of nearly 40 are required to show significant differences between schizophrenic and control groups [27].

Incidental findings: is MRI a too sensitive predictor of developing disease?

As many researchers who have performed MRI research studies in non-patient volunteers can attest the technique can at times be disconcertingly sensitive to asymptomatic pathology, also known as 'incidentalomas' [28]. For example Hartwigsen found in a research study which produced whole brain structural MRs that $19 \%$ of subjects had 'incidental findings' of which $10 \%$ were considered to be of potential clinical significance, including arteriovenous malformations, cavernomas and pituitary abnormalities.

In a meta review of 16 such studies by Morris [29] in which subjects without neurological symptoms were studied, prevalence of neoplastic brain lesions was $0.7 \%$, the incidence of which increased with age (thought mainly to be due to meningiomas with a $0.29 \%$ incidence). Other lesions considered clinically relevant (eg cysts, hydrocephalus, inflammatory lesions, Arnold Chiari malformations and vascular abnormalities) had a $2 \%$ prevalence with arachnoid cysts at $0.5 \%$ and aneurysms at $0.35 \%$. A range of other incidental findings were not included in the above figures because of their unknown role in causing symptoms and uncertainty regarding the need for intervention. Such conditions included white matter intensities (also called periventricular white matter objects or PVWOs), silent infarcts and brain microbleeds. They also did not focus on findings considered to be anatomical variants such as asymmetric ventricles and large cisterna magna.

On the face of it studies such as these would appear to be a diagnostic boon as they potentially reveal abnormalities which may be remediable even before symptoms appear. However the significance of such lesions has not yet been established and indeed many may never cause the patient any such symptoms. For example, as Morris [29] points out, most unruptured aneurysms have a low risk of haemorrhage, perhaps even lower than the risk from remedial surgery. Yano et al. [30] have shown that over $90 \%$ of asymptomatic meningiomas remain that way, and that $63 \%$ do not grow at all. Neurosurgery for such conditions is itself not without risk.

In its early years MRI was used most extensively in the brain and so most of the reported studies focus on incidental findings there. However studies in other parts of the body are appearing and indications are that prevalence of incidentalomas may be even greater. It has been found with MRI that the incidence of pancreatic cysts in the normal population is $13.5 \%$ [31]. Jensen [32] using MR enterography in patients with suspected Crohns Disease found unrelated and unsuspected extra-intestinal findings not related to CD in $20 \%$ of these patients. Though $0.7 \%$ of patients benefitted from subsequent examinations it led to unnecessary examinations in $3.2 \%$. In conclusion they were of the opinion that a substantial number of patients experienced unnecessary morbidity because of additional examinations of benign or normal conditions.

Recent technical developments have meant that new clinical systems can readily scan the whole body as opposed to restricting imaging to specific organs of interest. This clearly greatly increases the potential for finding incidentalomas. Hegenscheid [33] performed whole body scans on 200 healthy volunteers and found 431 pathological findings in $176(88 \%)$ of them. Of these the majority were considered benign but 45 (10.4\%) required further work up. Morin [34] found abnormalities in a quarter of 148 healthy normal volunteers, nearly $13 \%$ of which were considered to be of clinical significance.

Establishing the clinical significance of such incidental findings is critical not only to avoid unnecessary further investigations, themselves with associated morbidity and mortality, but also because of the impact on the patient obtaining insurance, getting or keeping a job, and also on their personal life and plans. Last but not least, there is the adverse effect on the patient's peace of mind. How many patients, on being told they have a lesion in their brain, would not subsequently become aware of, and suffer from, apparent symptoms?

What is lacking is an extensive long term follow up of incidental findings in MR to attempt to better establish their predictive significance, if any, and provide data to better inform the need for intervention.

Until such studies are performed there remains considerable concern about the growing number of commercial organisations who offer whole body screening to the 
asymptomatic (sometimes called the 'worried well') often using ionising radiation techniques such as $\mathrm{CT}$, but more latterly using MRI [28].

\section{Conclusions and outlook}

MRI has proven itself to be one of the most important diagnostic imaging modalities presently available. Its application in the field of prognosis and prediction has however been limited. There is certainly evidence of such abilities in psychiatric conditions and dementias but generally only on the basis of grouped data. Prognosis and prediction in the individual patient is restricted to specific conditions such as tumour evolution and the effects of therapies and, more acutely, in stroke. The plethora of parameters available from MRI and MRS scans do however offer hope for novel predictive models in the future. Combinations of these parameters, for example metabolite concentrations from MRS coupled with the volumetric data from MRI, may yield more sensitive prognostic and predictive indicators of subtle psychiatric disease in affected structures such as the hippocampus.

The technology of MR is continuing to rapidly evolve with the move to even higher magnetic fields allowing much more accurate estimates of many of the biomarkers described here. Coupled with this the use of many more radiofrequency coils simultaneously is allowing acquisitions to become almost real time allowing better temporal and spatial resolution. Additionally hybrid systems are becoming commercially available which combine both MR and positron emission tomography (PET) to more intimately relate morphology and function. The applicability of MR to prognosis and prediction can therefore only increase.

MRI has been shown to detect many incidental findings' both in the brain and with much higher frequency in the rest of the body. Until the prognostic import of these has been established with long term follow-up studies, great care should be taken in assigning any clinical significance to these 'incidentalomas'.

Acknowledgements I would like to thank my colleagues Prof. Donald Hadley, Dr. Gillian MacNaught and Dr. Krishna Dani for supplying some of the illustrations.

\section{References}

1. Hannemann J, Oosterkamp HM, Bosch CA, Velds A, Wessels LF, Loo C, et al. Changes in gene expression associated with response to neoadjuvant chemotherapy in breast cancer. J Clin Oncol. 2005;23:3331-42.

2. Fiorentino C, Berruti A, Bottini A, Bodini M, Brizzi MP, Brunelli A, et al. Accuracy of mammography and echography versus clinical palpation in the assessment of response to primary chemotherapy in breast cancer patients with operable disease. Breast Cancer Res Treat. 2001;69:143-51.

3. Loo CE, Teertstra J, Rodenhuis S, van de Vijver M, Hannemann J, Muller S, et al. Dynamic Contrast-Enhanced MRI for Prediction of Breast Cancer Response to Neoadjuvant Chemotherapy: Initial Results. AJR Am J Roentgenol. 2008;191:1331-8.

4. Knopp MV, Weiss E, Sinn HP, Mattern J, Junkermann H, Radeleff $\mathrm{J}$, et al. Pathophysiologic basis of contrast enhancement in breast tumors. J Magn Reson Imaging. 1999;10(3):260-6.

5. Cheung YC, Chen SC, Su MY, See LC, Hsueh S, Chang HK, et al. Monitoring the size and response of locally advanced breast cancers to neoadjuvant chemotherapy (weekly paclitaxel and epirubicin) with serial enhanced MRI. Breast Cancer Res Treat. 2003;78:51-8.

6. Dempsey MF, Condon BR, Hadley DM. Measurement of tumor "size" in recurrent malignant glioma: 1D, 2D, or 3D? AJNR Am J Neuroradiol. 2005;26(4):770-6.

7. Walker J, Bhullar N, Fallah-Rad N, Lytwyn M, Golian M, Fang T, et al. Role of three-dimensional echocardiography in breast cancer: comparison with two-dimensional echocardiography, multiple-gated acquisition scans, and cardiac magnetic resonance imaging. J Clin Oncol. 2010;28(21):3429-36.

8. Muir KW. Heterogeneity of stroke pathophysiology and neuroprotective clinical trial design. Stroke. 2002;33:1545-50.

9. Fiehler J, Foth M, Kucinski T, Knab R, von Bezold M, Weiller C, et al. Rother J Severe ADC decreases do not predict irreversible tissue damage in humans. Stroke. 2002;33:79-86.

10. Dani K, Santosh C, Brennan D, McCabe C, Holmes W, Condon B, et al. T2* Weighted Magnetic Resonance Imaging with Hyperoxia in Acute Ischemic Stroke. Ann Neurol. 2010;68(1):37-47.

11. Barnes J, Bartlett JW, van de Pol LA, Loy CT, Scahill RI, Frost C, et al. A meta-analysis of hippocampal atrophy rates in Alzheimer's disease. Neurobiol Aging. 2009;30(11):1711-23.

12. Tabrizi SJ, Scahill RI, Durr A, Roos RA, Leavitt BR, Jones R, et al. Biological and clinical changes in premanifest and early stage Huntington's disease in the TRACK-HD study: the 12-month longitudinal analysis. Lancet Neurol. 2011;10(1):31-42.

13. Ridha BH, Barnes J, Bartlett JW, Godbolt A, Pepple T, Rossor $\mathrm{MN}$, et al. Tracking atrophy progression in familial Alzheimer's disease: a serial MRI study. Lancet Neurol. 2006;5(10):828-34.

14. Condon B, Grant R, Hadley D, Lawrence A. Brain and intracranial cavity volumes: in vivo determination by MRI. Acta Neurol Scand. 1988;78:387-93.

15. Frisoni GB, Fox NC, Jack CR Jr, Scheltens P, Thompson PM. The clinical use of structural MRI in Alzheimer disease. Nat Rev Neurol. 2010;6(2):67-77.

16. Scheltens P, Leys D, Barkhof F, Huglo D, Weinstein HC, Vermersch P, et al. Atrophy of medial temporal lobes on MRI in "probable" Alzheimer's disease and normal ageing: diagnostic value and neuropsychological correlates. J Neurol Neurosurg Psychiatry. 1992;55(10):967-72.

17. Bouwman FH, Schoonenboom SN, van der Flier WM, van Elk EJ, Kok A, Barkhof F, et al. CSF biomarkers and medial temporal lobe atrophy predict dementia in mild cognitive impairment. Neurobiol Aging. 2007;28:1070-4.

18. Barkhof F, Filippi M, Miller DH, Scheltens P, Campi A. Comparison of MRI criteria at first presentation to predict conversion to clinically definite multiple sclerosis. Brain. 1997;120:2059-69.

19. Prosperini L, Gallo V, Petsas N, Borriello G, Pozzilli C. One-year MRI scan predicts clinical response to interferon beta in multiple sclerosis. Eur J Neurol. 2009;16(11):1202-9.

20. Petkau J, Reingold SC, Held U, Cutter GR, Fleming TR, Hughes $\mathrm{MD}$, et al. Magnetic resonance imaging as a surrogate outcome for multiple sclerosis relapses. Mult Scler. 2008;14(6):770-8. 
21. Chen CH, Ridler K, Suckling J, Williams S, Fu CH, Merlo-Pich $\mathrm{E}$, et al. Brain Imaging Correlates of Depressive Symptom Severity and Predictors of Symptom Improvement After Antidepressant Treatment. Biol Psychiatry. 2007;62:407-14.

22. Block W, Träber F, von Widdern O, Metten M, Schild H, Maier $\mathrm{W}$, et al. Proton MR spectroscopy of the hippocampus at $3 \mathrm{~T}$ in patients with unipolar major depressive disorder: correlates and predictors of treatment response. Int J Neuropsychopharmacol. 2009;12(3):415-22.

23. Kado H, Kimura H, Murata T, Nagata K, Kanno I. Depressive psychosis: clinical usefulness of MR spectroscopy data in predicting prognosis. Radiology. 2006;238(1):248-55.

24. Brugger S, Davis JM, Leucht S, Stone JM. Proton magnetic resonance spectroscopy and illness stage in schizophrenia-a systematic review and meta-analysis. Biol Psychiatry. 2011;69 (5):495-503.

25. Keshavan MS, Dick RM, Diwadkar VA, Montrose DM, Prasad KM, Stanley JA. Striatal metabolic alterations in non-psychotic adolescent offspring at risk for schizophrenia: a (1)H spectroscopy study. Schizophr Res. 2009;115(1):88-93.

26. Jessen F, Scherk H, Träber F, Theyson S, Berning J, Tepest R, et al. Proton magnetic resonance spectroscopy in subjects at risk for schizophrenia. Schizophr Res. 2006;87(1-3):81-8.

27. Steen RG, Hamer RM, Lieberman JA. Measurement of brain metabolites by $1 \mathrm{H}$ magnetic resonance spectroscopy in patients with schizophrenia: a systematic review and meta-analysis. Neuropsychopharmacology. 2005;30(11):1949-62.

28. Salman R, Whiteley WN, Warlow C. Screening using whole-body magnetic resonance imaging scanning: who wants an incidentaloma? J Med Screen. 2007;14(1):2-4.

29. Morris Z, Whiteley WN, Longstreth WT Jr, Weber F, Lee YC, Tsushima $\mathrm{Y}$, et al. Incidental findings on brain magnetic resonance imaging: systematic review and meta-analysis. BMJ. 2009;339: b3016.

30. Yano S, Kuratsu J, Kumamoto. Indications for surgery in patients with asymptomatic meningiomas based on an extensive experience. J. Neurosurg. 2008;105:538-43.

31. Lee KS, Sekhar A, Rofsky NM, Pedrosa I. Prevalence of incidental pancreatic cysts in the adult population on MR imaging. Am J Gastroenterol. 2010;105(9):2079-84.

32. Jensen MD, Nathan $T$, Kjeldsen J, Rafaelsen SR. Incidental findings at MRI-enterography in patients with suspected or known Crohn's disease. World J Gastroenterol. 2010;16(1):76-82.

33. Hegenscheid K, Kühn JP, Völzke H, Biffar R, Hosten N, Puls R. Whole-body magnetic resonance imaging of healthy volunteers: pilot study results from the population-based SHIP study. Rofo. 2009;181(8):748-59.

34. Morin SH, Cobbold JF, Lim AK, Eliahoo J, Thomas EL, Mehta $\mathrm{SR}$, et al. Incidental findings in healthy control research subjects using whole-body MRI. Eur J Radiol. 2009;72(3):529-33. 